\title{
Up-scaling Two-Phase Flow in Heterogeneous Reservoirs: Current Trends
}

\author{
V. Artus ${ }^{1}$ and B. Nœtinger ${ }^{1 *}$ \\ 1 Institut Français du Pétrole, Direction Ingéniérie de Réservoirs, \\ 1 et 4, avenue de Bois-Préau, 92852 Rueil-Malmaison Cedex - France \\ e-mail: vincent.artus@ifp.fr - benoit.noetinger@ifp.fr \\ * Corresponding author
}

\begin{abstract}
Résumé - Mise à l'échelle des écoulements diphasiques en réservoir hétérogènes, de nouvelles approches - Dans cet article, nous passons en revue les principales techniques de mise à l'échelle des écoulements diphasiques. Nous examinons à la fois les approches purement numériques dont le but est directement l'agrégation de maillage, et les approches physiques où l'on cherche la forme prise par les équations de transport, après une prise de moyenne sur le désordre éventuel du milieu poreux sous-jacent. Par une étude détaillée de la structure du front eau-huile, nous montrons en particulier que l'approche stochastique ne peut faire l'économie du couplage entre le champ de pression et l'évolution de la carte de saturation, et qu'il existe une interaction très forte entre l'hétérogénéité et la nature stable ou instable de l'écoulement. Cette interaction est responsable d'un changement qualitatif et quantitatif de la forme des équations à grande échelle, que toute technique de changement d'échelle se doit donc de respecter. Une fois ce problème résolu, on peut s'intéresser à une discrétisation optimale des modèles et des équations d'écoulement à grande échelle, permettant d'accélérer les simulations, et d'optimiser les études d'incertitudes de type Monte Carlo. Des exemples sont donnés dans des cas stratifiés, ou hétérogènes aléatoires.
\end{abstract}

\begin{abstract}
Up-scaling Two-Phase Flow in Heterogeneous Reservoirs: Current Trends - In this paper, we review the main upscaling techniques of two phase flow. Purely numerical fine to coarse gridding methods are investigated, as well as more physical approaches where the focus is on the algebraic form of the averaged transport equations. A detailed numerical and analytical study of the dynamics of the water-oil front shows that stochastic approaches cannot neglect the viscous coupling between the pressure and saturation. A very strong interaction exists between the heterogeneity and the stable or unstable character of the fluid flow displacement. This coupling is responsible of a qualitative and quantitative change of the form of the large scale equations, that must be accounted for by any upscaling procedure. Once this problem is solved, it is easier to devise optimal physically based discretisation procedures, that allows Monte-Carlo studies to be accelerated. Examples dealing with stratified or stochastic media will also be presented.
\end{abstract}




\section{NOMENCLATURE}

$\alpha \quad$ Frequency mode in y-direction

$\lambda$ Total mobility

$\gamma_{x} \quad$ Front variogram

$\mu_{o} \quad$ Oil viscosity

$\mu_{w} \quad$ Water viscosity

$\phi \quad$ Porosity

$c \quad$ Base state front velocity

$\widehat{C}_{K} \quad$ Fourier transform of the correlation function of the permeability field

$\widehat{C}_{x} \quad$ Fourier transform of the correlation structure of the front line

$f(S)$ Fractional flow

$h \quad$ Distance in $y$ direction between two points of the front

$K \quad$ Single-phase permeability

$\delta K_{\alpha} \quad$ Single-phase permeability perturbation mode $\alpha$

$k_{r o} \quad$ Oil relative permeability

$k_{r w} \quad$ Water relative permeability

$M_{f} \quad$ Total mobility ratio evaluated across the front

$p \quad$ Pressure

$q \quad$ Frequency mode in y-direction

$S \quad$ Saturation

$S_{f} \quad$ Front saturation

$S_{\text {min }}$ Water irreducible saturation

$t \quad$ Time

$u_{0} \quad$ Base state total filtration velocity

$\delta u_{b \alpha x}$ Total velocity perturbation mode $\alpha$ in $\mathrm{x}$-direction induced by heterogeneity

$x_{0} \quad$ Base state front position in $\mathrm{x}$-direction

$\delta x_{\alpha} \quad$ Perturbation mode $\alpha$ of front line

$Y \quad$ Logarithm of the single-phase permeability

$\delta Y_{\alpha} \quad$ Perturbation mode $\alpha$ of $Y$

$\delta \boldsymbol{u}_{\boldsymbol{a}} \quad$ Total velocity perturbation vector induced by viscous effects

$\delta \boldsymbol{u}_{\boldsymbol{b}} \quad$ Total velocity perturbation vector induced by heterogeneity

$\boldsymbol{u}$ Total filtration velocity vector

$\boldsymbol{x} \quad$ Location vector

\section{INTRODUCTION}

From early numerical reservoir models which appeared in the sixties, involving several hundreds of cells, to actual current million-cell stochastic models, grids used to describe reservoirs heterogeneities have always been by far too large and expensive in terms of computational requirements for flow simulations. Upscaling from a detailed reservoir model to a coarse grid model is hence still a challenging issue for reservoir engineers, in order to speed up flow simulations. However, the meaning of upscaling has partially been affected by the emergence of stochastic reservoir modeling techniques, and previous upscaling methods can be useless within a stochastic framework. For example, when dealing with an infinite number of equally likely reservoir models, should the upscaling process be able to lead to the same simulation results for each realisation of the permeability field, or should it only allow us to quickly derive our uncertainty on the recovery curve with respect to the uncertainty on the field and on the production scenario?

We review some of the main existing approaches in upscaling of two-phase flow and we propose a stochastic model allowing the dynamics of the front to be described in heterogeneous porous media. We show that the upscaling process should first involve a physical step to relate local flow properties to large-scale flow properties. This step is independant of the size of gridblocks and of the numerical scheme used for flow simulations, as we deal mainly with the underlying continuous equations. The numerical problem of gridding the large-scale model should be investigated in a subsequent step. A study of the physical problem shows that viscous coupling is a crucial phenomenon that controls the emergence of different large-scale flow regimes. This leads us to propose a new stochastic model to describe the dynamics of the front in heterogeneous media. This promising model gives answers to the physical problem and should be helpful to optimize upscaling. An application to stratified media shows the emergence of different flow regimes, depending on the frontal mobility ratio. The front may move at the same uniform velocity in some layers, that we called hydrodynamic layers. This phenomenon allows an optimal grouping of the original geological layers, that takes into account their ordering.

In the case of an isotropic permeability map, we are able to relate the statistical structure of the water-oil front (mean, covariance function) to that of the underlying permeability map, and to the fluid properties, mainly the total mobility ratio evaluated at the front. In the stable case, this leads to the formation of a quasi stationary front. Coming back to a local Eulerian description shows that this stationary solution can correspond to the result of a competition between the relaxation process corresponding to the stabilitisation of the front, and the velocity perturbation induced by the heterogeneities. This indicates that a large scale equation having the form of a classical two-phase flow saturation transport equation could work, with a suitable renormalisation of the fractional flow term, and with the addition of a diphasic dispersion term.

\section{NUMERICAL APPROACH}

Most practical solutions for upscaling two-phase flow in heterogeneous media aim at computing the properties of a coarse grid in such a way that the simulation on this grid gives results as close as possible from the results of a simulation on the reference fine grid. The nature of flow equations is 
supposed to remain the same from the fine grid to the coarse grid. For example, the equations for the saturation of the injected fluid $S$ and the pressure $p$ are locally given by:

$$
\begin{aligned}
\nabla \cdot\left(\lambda_{t} \cdot \nabla p\right) & =0 \\
\phi \frac{\partial S}{\partial t}+\nabla \cdot(\boldsymbol{u} f(S)) & =0
\end{aligned}
$$

where $\phi$ is the porosity, $\lambda_{t}(S)$ is the total mobility, $\boldsymbol{u}$ is the velocity field and $f(S)$ is the fractional flow. Upscaling computes effective properties associated with the coarse gridblocks:

$$
\begin{array}{r}
\nabla \cdot\left(\overline{\lambda_{t}} \cdot \nabla \bar{p}\right)=0 \\
\bar{\phi} \frac{\partial \bar{S}}{\partial t}+\nabla \cdot(\overline{\boldsymbol{u}} \cdot \bar{f}(\bar{S}))=0
\end{array}
$$

These derived effective properties should hence solve two problems in a single step: first, accounting for the average effects of the underlying heterogeneity on the flow at the scale of a coarse gridblock, and second, accounting for the numerical dispersion associated with using a coarser grid.

\subsection{Pseudofunctions}

\subsubsection{Stratified Media}

Robust solutions for this problem have been given in stratified media. When the assumption of no-communication between all layers is verified, a famous method from Stiles (1949), and Dykstra and Parsons (1950), allows us to simply derive the mean flow properties in the transverse direction. This powerful method reduces the problem to one dimension but requires very low communication in the reservoir, which is not always the case. In the opposite case, when excellent communication exists in the direction orthogonal to the layers, the hypothesis of transverse equilibrium may be invoked. Two main kinds of transverse equilibrium can be met: When capillary or gravity forces are predominant compared with viscous forces, the segregation of fluids is fast while flooding and the saturation field can be derived anywhere. Static pseudofunctions can hence be computed in the transverse direction, relying on the assumption that both gravity and capillary forces dominate, (Coats et al. 1967; Killough and Foster 1979), that gravity forces only dominate (Martin, 1968; Coats et al., 1971) or that capillary forces dominate (Yokoyama and Lake, 1981). These methods are robust but it is difficult to verify if the equilibrium hypothesis are actually met in the reservoir. Criteria allowing determination of whether the equilibrium state is likely or not are given in Dake (1978) and Ahmadi (1992).

Another situation is met when viscous forces are predominant compared with other forces, and when the communication between all layers is assumed to be perfect (Hearn, 1971; Simon and Koederitz, 1982). Under this assumption, a viscous transverse equilibrium emerges, and the saturation field moves in the medium such that no transverse pressure gradients exist. It can be shown that under this assumption, each layer is flooded at a constant rate. These rates depend on the fluid viscosities and on the permeability of the layer. An effective fractional flow in the transverse direction can hence be derived for each mean saturation. The average relative permeabilities can be computed from this effective fractional flow.

Transverse equilibrium methods give accurate results as they take into account the physical mechanisms involved while flooding. However, they assume extreme conditions regarding the communication between all layers, that may be rarely met in actual reservoirs. When dealing with dynamic flows or non-stratified reservoirs, other methods must hence be used.

\subsubsection{Dynamic Pseudofunctions}

When transverse equilibrium is not met in the reservoir, the saturation map cannot be easily derived and one should rely on the results of a simulation on the fine grid to derive mean properties called dynamic pseudofunctions. This has been introduced by Jacks et al. (1973), to coarsen the grid in the vertical direction and has been extended by Kyte and Berry (1975), to coarsen the grid in all directions, taking into account the numerical dispersion. The use of dynamic pseudo-functions for upscaling gave rise to many methods. Detailed reviews can be found in Stone (1991), Ahmadi, (1992) and Archer, (1996).

The simulation is first performed on the fine grid. The mean saturation, viscosities and densities in each coarse block are hence computed by averaging the values of the underlying fine gridblocks. Two main approaches are possible to derive the pseudo relative permeabilities associated with the mean saturations. A first solution consists in defining average pressure gradients on the coarse gridblocks (Jacks et al., 1973; Kyte and Berry, 1975; Kossack et al., 1990; Guzman et al., 1999) and introducing them into Darcy's law to derive pseudo relative permeabilities so that the fluxes across a coarse block are conserved from the fine grid to the coarse grid. A second solution consists in directly computing the pseudo-fractional flows on the coarse blocks and deriving the pseudo relative permeabilities from these fractional flows in order to conserve the fluxes from one grid to another (Stone, 1991; Hewett and Behrens, 1991).

As the derived pseudofunctions account both for the effects of heterogeneity and for the control of numerical dispersion, the physical meaning of the properties derived on the coarse grid is lost (Chang and Mohanty, 1997, Durlofsky, 1997). Pseudo relative permeability functions are often affected by erratic variations, and can even be negative with Kyte and Berry's method! The loss of physical meaning due to the numerical dispersion control can be illustrated with a homogeneous medium. Coarsening the fine grid of such a medium with pseudofunctions will lead to a heterogeneous coarse grid. 
Pseudofunctions are only valid for the particular case of fine grid and production scenario used for the reference simulation. One set of pseudofunctions is derived for each coarse block. Some practical issues are hence raised when trying to use pseudofunctions with large models, for which a complete simulation on the fine grid is impossible (Barker and Thibeau, 1992). In these situations, the reference simulation is performed on a representative part of the grid and results are interpolated on the whole grid. This is likely to give erroneous results, due to the dependence of pseudofunctions to the boundary conditions and to the position of blocks in the coarse grid. Moreover, a complete sensitivity study with respect to the production scenarios should involve a computation of new pseudofunctions for each new tested scenario, which is very time-consuming in practice.

\subsection{Dual Mesh Methods}

Another very promising approach consists in using a coarse grid for computing the pressure field, while moving the saturations on the reference fine grid by an adapted downscaling step. This methods takes advantage of the fact that in many reservoir simulators, an IMPES resolution scheme is used: the pressure equation is discretised using an implicit scheme, while the saturation map is up-dated using an explicit time scheme, so they can be solved separatly and iteratively. As the pressure equation is elliptic, a coarser grid can be used to solve it. Introduced in a monophasic context by Rame and Killough 1991, this method has been extended to two-phase flow by Guerillot and Verdière, 1996 and 1996, Verdière and Vignal, 1998, Guedes and Schiozer, 1999, Gautier et al, 1999.

These methods are based on the following steps. (1) The saturation map is given on the fine grid. (2) The saturations are averaged to give the saturation map on the coarse grid. (3) The pressure and velocity fields are computed on the coarse grid. (4) The velocity field in the fine blocs underlying the coarse blocs is computed by a kind of "downscaling" step, assuming the flux into a fine block located at the boundary of a coarse block to be proportionnal to the block's transmissivity. (5) The saturations are updated on the fine grid.

This method was extended to the finite elements context by Arbogast and Bryant, 2001. It is very attractive, as it allows robust and fast predictions. However, the "downscaling" step from the coarse to fine grid is still relying on the assumption that fluxes are proportionnal to the transmissivity, which is not always the case. Some theoretical work on the fluxes is hence still required.

Moreover, this method is designed to upgrid from a single detailed realisation of the reservoir to a coarse model, and does not take into acount the stochastic nature of the reservoir description. In order to handle uncertainty, the upscaling step should be performed for every realisation of the permeability field.

\subsection{Non-Uniform Coarsening}

Another approach takes advantage of the possibilities offered by the developpement of nonuniform gridding techniques (Aziz, 1993). This allows high resolution in complex zones of the reservoir (vicinity of wells, faults, pinchouts, ...) to be maintained, while using coarser cells where high resolution is not required. The geological and structural properties of the field are thus conserved.

The main problem of this approach consists in correctly deriving the fluxes in the reservoir (Ponting, 1989, Peaceman, 1996) with appropriate numerical schemes. Moreover, the coarse grid generation is mainly based on static considerations, without paying attention to the physical problem of dynamic two-phase flow. For example, uplayering techniques (Li et al, 1995, Stern and Dawson, 1999, Li and Beckner, 2000) search for an optimal grouping of layers in the vertical direction, keeping only the main permeability contrasts.

Streamtubes techniques allow the fluxes to be evaluated and the grid shape to be optimised with respect to these fluxes, with a resolution of the pressure field on the fine grid (Prevost, 2001 and 2002). High velocity zones are then finely gridded, while the grid is coarsened in slower zones. This introduces dynamic considerations in the gridding step. However, the underlying assumption is that the fluxes in the reservoir and the shape of the streamtubes do not dramatically change while fluids are flowing, which could be invalid when high mobility contrasts exist between the two fluids: this will be one conclusion of this paper.

This approach to the upscaling problem, neglecting some part of the information by localy coarsening the grid, is based on the following essential consideration: the uncertainty on the simulation result due to the uncertainty on the model itself is much more dramatic than the error associated with upscaling (Glimm et al, 2003). As a consequence, it is only worth retaining a good precision in the dominant zones of the reservoir, by adapting the gridding.

\subsection{The Need for Another Description}

Actually, when the reservoir description is stochastic, it is not necessary to use an upscaling method that reproduces exactly the results of the fine grid, and a method allowing the mean and the variance of the recovery curve to be predicted may be adequate (Nœtinger, 2000). Ideally, the model could even be generated directly at the desired scale, and refined by iterations during the caracterization study (Gorell and Bassett, 2001).

We have seen that changes in the physics of the flow from one scale to another is often neglected by upscaling methods. The problem of upscaling reservoir models is currently seen as the process of directly coarsening the reference grid, using the same set of parameters like porosities, permeabilities and so on. The parameters on the coarse grid hence take effective 
values that account for both the effects of the underlying heterogeneity and the effects of numerical dispersion associated with the size of the new cells.

Here, we split the upscaling problem into two separate steps. We propose to first derive the average equations for two-phase flow at large scale. From the stochastic description of the reservoir at fine scale, using a given set of fine scale random properties, this first step allows a large scale model to be derived with new appropriate and average parameters at large scale. These parameters should account for the average effects of the underlying heterogeneity (for example, see the emergence of a macrodispersive term in the large-scale saturation equation for single-phase flow in heterogeneous media, Gelhar, 1983).

Once this first, physical step has been solved, the numerical problem of gridding the large-scale model with coarse cells can be investigated. We believe that a correct physical, continuous model at large scale will be helpful to choose the optimal grid during the numerical step. In our study, we only investigated the physical step, keeping the numerical problem for subsequent research.

\section{PHYSICAL APPROACH}

\subsection{Flow Regimes at Large Scale}

The regime of a two-phase displacement in a heterogeneous porous medium is strongly dependant on the properties of the flowing fluids (viscosities, densities), the properties of the field (variance, corelation function, ...) and the displacement velocity. These regimes can be characterized by the evolution of the front line between the two fluids while flooding.

Lenormand, 1996 describes 3 main flow regimes for twophase piston-like displacements in heterogeneous media, without capillarity or gravity effects. (1) When the fluid in place is less viscous than the injected fluid, and when the correlation length $l_{c}$ of the permeability field is much smaller than the field scale $L$, the flow regime is macrodispersive. In this case, the perturbations of the front line, induced by the heterogeneity, remain small while flooding; (2) When $l_{c} / L$ is still small but the injected fluid is less viscous than the fluid in place, the flow regime becomes viscous unstable: perturbations of the front line are amplified while flooding and the saturation map shows viscous fingers, the geometry of which is slightly dependant on the permeability field; (3) When the injected fluid is more viscous than the fluid in place but $l_{c} / L$ tends toward 1 , the heterogeneity dominates the flow and the regime is said to be channeling. Between those three main regimes, intermediate regimes can occur. A convenient tool is to describe these different regimes by a phase diagram, analogous to those employed in thermodynamics. Of course, a lot of other parameters could have been introduced, such as densities, injection rates, different correlation laws for the permeability field, ..., leading to increasingly complex diagrams (Genty, 1999, Furtado and Pereira, 2002).
The large scale saturation profiles derived from twophase displacements in heterogeneous media exhibit different behaviors, depending on the flow regime. In particular the time evolution of the mixing zone at large scale differs from one regime to another (Lenormand, 1996, Furtado and Pereira, 2002). Hence we can suppose that the nature of the flow equation at large scale must change, to be able to describe these different qualitative flow regime. Correct flow equations at large scale should hence be derived to get a correct description when upscaling. Solutions have been proposed in the viscous unstable case by, among others, Koval, 1963, Todd and Longstaff, 1972, or Blunt and Christie, 1993. Another approach unifing the three regimes with a single model has been proposed by Lenormand, 1996.

The main advantage of these considerations is to show that depending on the value of several key parameters at the local scale, the flow at large scale will converge towards some different flow regimes, and will be well-described by new key parameters of the new scale. However, the proposed models for flow equations at large scale are mainly empirical, and theoretical works still remain necessary, using stochastic methods.

\subsection{Stochastic Models}

The stochastic approach allows the uncertainty on the permeability field to be dealt with. The aim is to derive the statistics of flow properties from the statistics of the permeability field. For example, one can try to get the equation of the mean saturation from properties of the local equation, using the statistics of the heterogeneous permeability field as data.

Considerable work has been undertaken for monophasic or tracer flows, for example by Dagan, 1989 and Gelhar, 1993. However, few explicit answers have been given for two-phase flows, mostly because of the coupling between the saturation field and the velocity field, that induces timedependant correlation terms between all variables. Most stochastic work for two-phase flow focus on steady-state (Ekrann and Aasen, 2000) or quasi-static flows (Quintard and Whitaker, 1988). When dealing with transient flow, the proposed solutions neglect the influence of viscous coupling.

Let us consider a waterflood in a heterogeneous reservoir, without capillary or gravity forces. The saturation $S(\vec{x}, t)$ and the pressure $p(\mathbf{x}, t)$ are locally given by the following equations:

$$
\begin{gathered}
\phi \frac{\partial S}{\partial t}+\nabla \cdot(f(S) \mathbf{u})=0 \\
\nabla \cdot(\lambda(S) \nabla p)=0
\end{gathered}
$$

where $\mathbf{x}$ is the location vector, $f(S)$ is the fractional flow, and $\mathbf{u}(\mathbf{x}, t)$ is the total filtration velocity, given by the two-phase extension of single-phase Darcy's law:

$$
\mathbf{u}=-\lambda \nabla p
$$


and $\lambda(S)$ is the total mobility term, given by:

$$
\lambda(S)=K\left(\frac{k_{r w}(S)}{\mu_{w}}+\frac{k_{r o}(S)}{\mu_{o}}\right)
$$

where $k_{r w}$ and $k_{r o}$ are the relative permeability curves for water and oil. As $\lambda$ depends on $S$, we see that Equation (5) and Equation (6) are coupled. This non-linearity is responsible for the correlations that appear between all variables. By defining all variables as a sum of their mean (-) plus a perturbation $(\delta$.), expanding the saturation equation to first order (assuming that $\sigma_{\ln (K)}$ is small) and averaging the expanded equation, the following equation for the mean saturation can be obtained (Langlo and Espedal, 1994):

$$
\phi \frac{\partial \bar{S}}{\partial t}+\nabla \cdot(f(\bar{S}) \overline{\mathbf{u}})+\nabla \cdot\left(\frac{d f}{d \bar{S}} \overline{\delta S \delta \mathbf{u}}+\frac{1}{2} \frac{d^{2} f}{d \bar{S}^{2}} \overline{\delta S \delta S} \mathbf{u}\right)=0
$$

We see from Equation (9) that a dispersive flux appears in the mean equation, due to the correlation terms. The extension of this equation to cases where gravity or capillarity are taken into account (Darman et al, 2000) induces other correlation terms. When working with transient flows, the saturation map is time-dependant and the coupling induces a timedependancy of all variables, so the correlation terms also depend on time. This does not occur with single-phase flows, for which the pressure and velocity fields do not evolve while flooding.

These correlation terms can have a significant impact (Durlofsky, 1997) on the large-scale flow, although their analytical form is difficult to derive. By averaging products of perturbations, one can obtain a system of equations for higher statistical moments (Jarman, 2000, Zhang, 2001). The main issue with this approach consists in finding a correct closure assumption to derive a practical solution. Without relying on severe assumptions, the numerical resolution of such a large system is a difficult task for two-phase flows.

The classical assumption made then consists in neglecting the time-dependancy of the statistical properties of the velocity field. Under this assumption, the covariance terms do not depend on time and $\overline{\delta \mathbf{u} \delta \mathbf{u}}$ can be found from the results of a tracer case (Rubin, 1990). As $\overline{\delta S \delta \mathbf{u}}$ and $\overline{\delta S \delta S}$ can be related to $\overline{\delta \mathbf{u} \delta \mathbf{u}}$, this leads in the Eulerian context to a macrodispersion expression for the saturation equation at large-scale (Langlo and Espedal, 1994):

$$
\frac{\partial \bar{S}}{\partial t}+\frac{\partial}{\partial x}(\bar{f}(\bar{S}) u)-\frac{\partial}{\partial x}\left(\frac{d \bar{f}\left(S_{f}\right)^{2}}{d \bar{S}} u \sigma_{Y}^{2} I \frac{\partial \bar{S}}{\partial x}\right)=0
$$

In this model, the large scale convective fractional flow used is simply proportionnal to the saturation value in the mixing zone, $\bar{S}<S_{f}$. As a consequence, this convective fractional flow cannot tend to build a front and the mixing zone will spread as $t^{1 / 2}$ under the effect of the macrodispersive term. Only one regime is hence predicted by this model, whatever the viscosities of the two fluids.
In the Lagrangian approach, the "no coupling" assumption consists in working with fixed streamlines. Using a statistical analysis of streamlines in heterogeneous media, and taking into account the hyperbolic displacement of saturations along the streamlines, a Lagrangian description can be obtained (Dagan et al, 1996a and 1996b, Zhang et al 1999). This approach predicts that the variance of the front line grows as $t^{1 / 2}$, whatever the viscosity values of the two fluids. Only one regime is hence predicted, similar to a tracer test. We have shown in a previous paper (Artus and Nœtinger, 2002) that the assumption of a fixed velocity field cannot lead to accurate predictions. The velocity field's updating due to viscous coupling plays a crucial role in the evolution of saturation maps, as it induces a crossflow that leads to different types of regimes, depending on the mobility ratio. In particular, when the injected fluid is less mobile than the fluid in place, the viscous coupling induces a crossflow that stabilizes the water-oil front: fingers induced by the heterogeneity cannot grow with time. On the contrary, when the injected fluid is more mobile than the fluid in place, the viscous coupling tends to favorize fingers: digitations induced by the heterogeneity continuously grow with time, in a similar way to the Saffman-Taylor (1952) unstability. Results derived under the assumption of no coupling cannot predict these behaviors and are closer to the results of a tracer test.

It is thus essential to take into account the viscous coupling to get a correct large-scale model of two-phase flows in heterogeneous porous media. For deterministic upscaling, the corelations induced by viscous coupling can be numerically computed from fine-scale simulations and included in the description (Darman et al 2000 and 2002). However, this cannot give access to a physical understanding of all mechanisms involved in a homogenisation step.

\section{A MODEL FOR THE INTERACTION BETWEEN HETEROGENEITY AND VISCOUS EFFECTS}

\subsection{Viscous Effects}

The main mechanism leading to different regimes seems to be the interaction between the heterogeneity and the viscous effects (Furtado and Pereira, 2002). The perturbations of the saturation map induced by the heterogeneity trigger the viscous effects. These viscous effects then interact with the heterogeneity while flooding and lead to the different regimes identified in the previous section.

Let us consider a homogeneous medium, and imagine that saturation planes are, for some reason, slightly deformed around their base-state position. For exemple, at a particular time $t$, the water-oil front is deformed around its base-state positon $x_{0}(t)$ :

$$
x_{0}(t)=u_{0} \frac{f^{\prime}\left(S_{f}\right)}{\phi} t
$$




$$
x(y, t)=x_{0}(t)+\frac{1}{2 \pi} \int_{-\infty}^{+\infty} \delta x_{\alpha}(t) e^{i \alpha y} d \alpha
$$

As this perturbes the saturation map, the pressure and velocity maps will also be perturbed because of the viscous coupling via $\lambda_{t}(S)$. These velocity perturbations will in turn play a role in the development of the saturation perturbations. This problem has been widely studied. King and Dunayevsky, 1989, have shown that, due to the viscous coupling, the position perturbation modes for the front in the homogeneous medium follow the following equation:

$$
\frac{\partial \delta x_{\alpha}}{\partial t}=c|\alpha| \frac{M_{f}-1}{M_{f}+1} \delta x_{\alpha}
$$

where:

$$
c=u_{0} \frac{f^{\prime}\left(S_{f}\right)}{\phi}
$$

is the velocity of the front in the base-state, and $M_{f}$ is the total mobility ratio evaluated accross the front:

$$
M_{f}=\frac{\lambda\left(S_{f}\right)}{\lambda\left(S_{\min }\right)}=\frac{\mu_{o} k_{r w}\left(S_{f}\right)+\mu_{w} k_{r o}\left(S_{f}\right)}{\mu_{o} k_{r w}\left(S_{\min }\right)+\mu_{w} k_{r o}\left(S_{\min }\right)}
$$

where $S_{\min }$ is the irreducible water saturation.

The stability of a waterflooding in a homogeneous medium hence depends on $M_{f}$. Any perturbation in the position of the front will be amplified while flooding if $M_{f}>1$, leading to an unstable flow. On the contrary, the perturbations will be damped while flooding if $M_{f}<1$ and the flood will remain stable. A critical regime occurs for $M_{f}=1$. A waterflooding can then exhibit a stable behavior, even if the injected fluid is less viscous than the fluid in place, because the relative permeability curves can be such that $M_{f}<1$ even for higher end-point viscosity ratios. For example, when the relative permeability curves are Coreytype with exponent of $2, M_{f}<1$ corresponds to $\frac{\mu_{o}}{\mu_{w}}<3$.

\subsection{Heterogeneous Media}

Let us now consider a heterogeneous field. The absolute permeability perturbations induce perturbations on the saturation, pressure and velocity fields. The evolution of saturation perturbations will then be driven by two contributions: the velocity fluctuations due to the heterogeneous permeability field, and the velocity field updating due to the viscous coupling.

Using a first-order expansion, we can say that a local velocity perturbation $\delta \mathbf{u}(x, y, t)$ is the sum of two contributions $\delta \mathbf{u}_{\mathbf{a}}(x, y, t)$ and $\delta \mathbf{u}_{\mathbf{b}}(x, y, t)$ :

$$
\delta \mathbf{u}(x, y, t)=\delta \mathbf{u}_{\mathbf{a}}(x, y, t)+\delta \mathbf{u}_{\mathbf{b}}(x, y, t)
$$

The first term, $\delta \mathbf{u}_{\mathbf{a}}$, is due to the fluctuations of the saturation planes around their mean position, in a homogeneous media. It expresses the fluctuations due to the contribution of the viscous coupling. The second term, $\delta \mathbf{u}_{\mathbf{b}}$ is due to the influence of the perturbations of the permeability field with a saturation map given by the base-state. It expresses the velocity perturbations directly due to the heterogeneity, and remains to be evaluated.

From Equation (13) and Equation (16), we can see that the evolution of a particular position perturbation mode of the front is given by:

$$
\frac{\partial \delta x_{\alpha}}{\partial t}=c|\alpha| \frac{M_{f}-1}{M_{f}+1} \delta x_{\alpha}+\frac{f^{\prime}\left(S_{f}\right)}{\phi} \delta u_{b \alpha x}
$$

This model allows an understanding of the interaction between heterogeneity and viscous effects. The heterogeneity of the permeability field induces perturbations $\delta \mathbf{u}_{\mathbf{b}}$ of the total filtration velocity field. These velocity perturbations modify the shape of the front by inducing front fluctuations $\delta x$ : this triggers the viscous effects (first right-hand side term in Equation (17)). When $M_{f}<1$, the mobility ratio across the front is favorable and viscous effects will be in competition with the perturbing effects of heterogeneity. An equilibrium is then likely to emerge between these two effects. When $M_{f}>1$, the mobility ratio across the front is unfavorable and viscous effects will amplify the perturbating effects of heterogeneity: an unstable regime occurs, and viscous fingering rapidly dominates the flow.

This model shows how the interaction between heterogeity and viscous effects leads to different regimes. Depending on the structure of the permeability field, different conclusions can be given on the front and on the way to derive a large-scale information.

\subsubsection{Stratified Media}

From a first-order development of Darcy's law, an xcomponent velocity perturbation mode in a stratified medium with a base-state saturation map is given by:

$$
\delta u_{b \alpha x}=\frac{u_{0}}{K_{0}} \delta K_{\alpha}
$$

When $M_{f}<1$, Artus et al, 2003 shows that each perturbation mode will reach a limit given by:

$$
\lim _{t \rightarrow+\infty} \delta x_{\alpha}(t)=-\frac{1}{|\alpha|} \frac{M_{f}+1}{M_{f}-1} \frac{\delta K_{\alpha}}{K_{0}}
$$

The stability of the process when $M_{f}<1$ hence lead to a stationary front. The amplitude of perturbations reaches a limit, when the crossflow induced by viscous effects is sufficient to compensate for permeability contrasts. An equilibrium is then reached. By using an inverse Fourier transform, we can obtain the form of the stationary front when the equibrium is reached:

$$
\delta x(y)=-\frac{c}{2 \pi A} \int_{-\infty}^{+\infty} \frac{\delta Y_{\alpha}}{|\alpha|} e^{i \alpha y} d \alpha
$$


with:

$$
A=c \frac{M_{f}-1}{M_{f}+1}
$$

The emergence of a stationary front when the mobility ratio is favorable is an essential information when choosing an upscaling method. As the width of the front does not grow while flooding, the loss of information is controlled when upscaling all layers in a single one. To first order, our model is able to relate the shape of the front to the permeability field. Accurate upscaling, downscaling and filtering are hence possible. Using this information can also help to directly generate media at a relevant scale.

However, a stationary front across the whole width of the medium is only possible when permeability contrasts are small and the mobility ratio can compensate for these contrasts. When high contrasts exist, the stratified medium is divided in several sub-units or "hydrodynamic layers" in which the front is stationary (Nœtinger et Artus, 2003, Artus, 2003). In these zones, the front travels at constant but different speeds. The hydrodynamic layers can be separated by non-stationary zones. As the front is stationary in the hydrodynamic layers, the underlying geological layers can be averaged into a single coarse layer with a controlled loss of information. The hydrodynamic layers should hence be identified and independently coarsened, providing a good starting point for non-uniform upscaling of stratified media (Fig. 1). As the building of stationary fronts depends on the value of the mobility ratio across the front and on permeability contrasts in the medium, the number and the position of hydrodynamic layers in a given medium will depend on the viscosity of the two fluids.

\subsubsection{Isotropic Media}

In isotropic media, the velocity perturbation $\delta u_{b}$ induced by the heterogeneity is quite difficult to derive. When $M_{f}<1$, Notinger et al. (2003) have shown that an equilibrium is reached between the permanent excitation due to the heterogeneity and the stabilizing viscous effects. This equilibrium induces a stationary behavior of the front, in a statistical sense. Let us define the corelation structure of the front, in the spectral domain, by :

$$
\widehat{C}_{x}(\alpha, t)=\overline{\delta x_{\alpha}(t) \delta x_{\alpha}(t)}
$$

and the variogram of the front by:

$$
\gamma_{x}(h, t)=\frac{1}{2 \pi} \int\left(\widehat{C}_{x}(\alpha, t)-\hat{C}_{x}(\alpha, t) e^{i \alpha h}\right) d \alpha
$$

The variogram for long times reaches a long-time stationary limit given by (see Notinger et al (2003) for technical details):

$$
\gamma_{x}(h)=\left(\frac{1}{4 \pi}\right)^{2} \frac{c^{2}}{\bar{K}^{2}} \iint \frac{\widehat{C}_{K}(\alpha, q)}{(A \alpha)^{2}+(q c)^{2}}\left(1-e^{i \alpha h}\right) d q d \alpha
$$

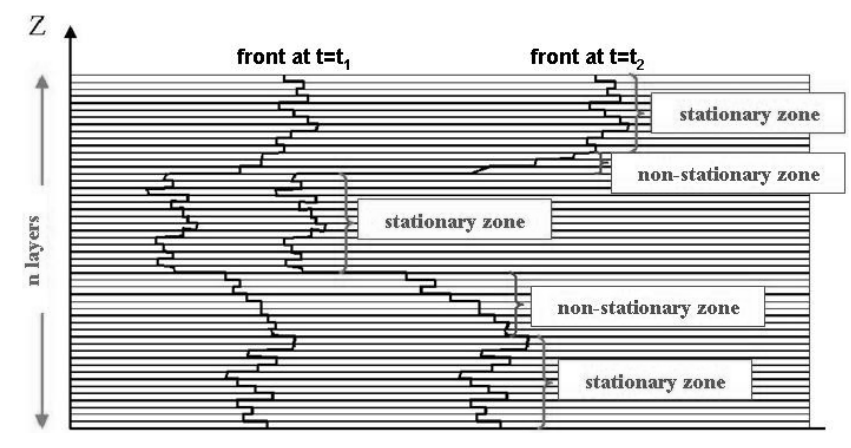

(a) Stratified input model.

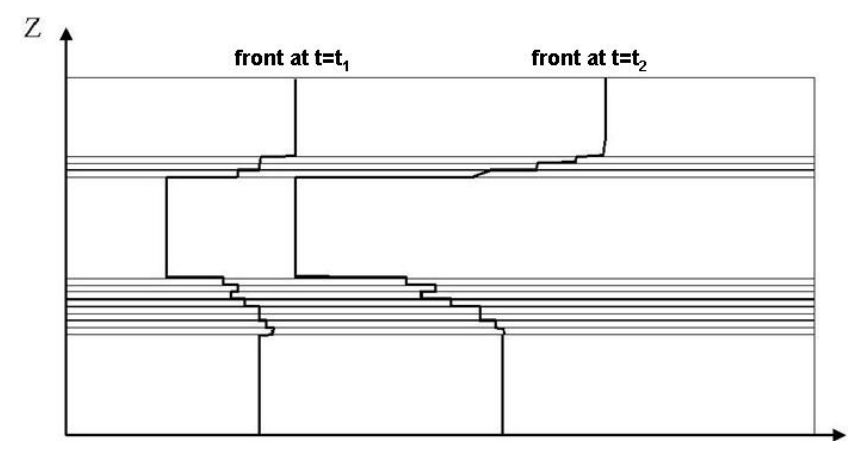

(b) Coarse model.

Figure 1

Conceptual model of grid coarsening using the concept of hydrodynamic layers where the front is stationnary.

where $\widehat{C}_{K}(\alpha, q)$ is the correlation function of the permeability field in the spectral domain.

From Equation (24), we observe that the flow pattern and the viscous term $A$ create an anisotropy on the front line. From this expression, one can derive directly the variogram of the front, knowing the covariance of the underlying permeability field, when the mobility ratio is favorable, without relying on Monte-Carlo simulations.

In order to illustrate this theoretical result, we computed the variogram of the front line from Monte-Carlo simulations on $200 \times 200$ cells heterogeneous reservoir models, with permeameter boundary conditions (Nœtinger and Artus, 2004). The absolute permeability fields were heterogeneous with variance $\sigma_{\ln (K)}^{2}=0.1$ and exponential correlation structure. The relative permeability curves were quadratic functions of the saturation, and were uniform in the media. The viscosity of the injected fluid was $\mu_{2}=10$ and the viscosity of the fluid in place was $\mu_{1}=1$, so that a stationary front occurs. The evolution of the variogram of the front with time is illustrated on Figure 2. On this figure, we clearly see that the variogram tends toward a long-time limit. We can also verify the slow logarithmic divergence of the variogram for 


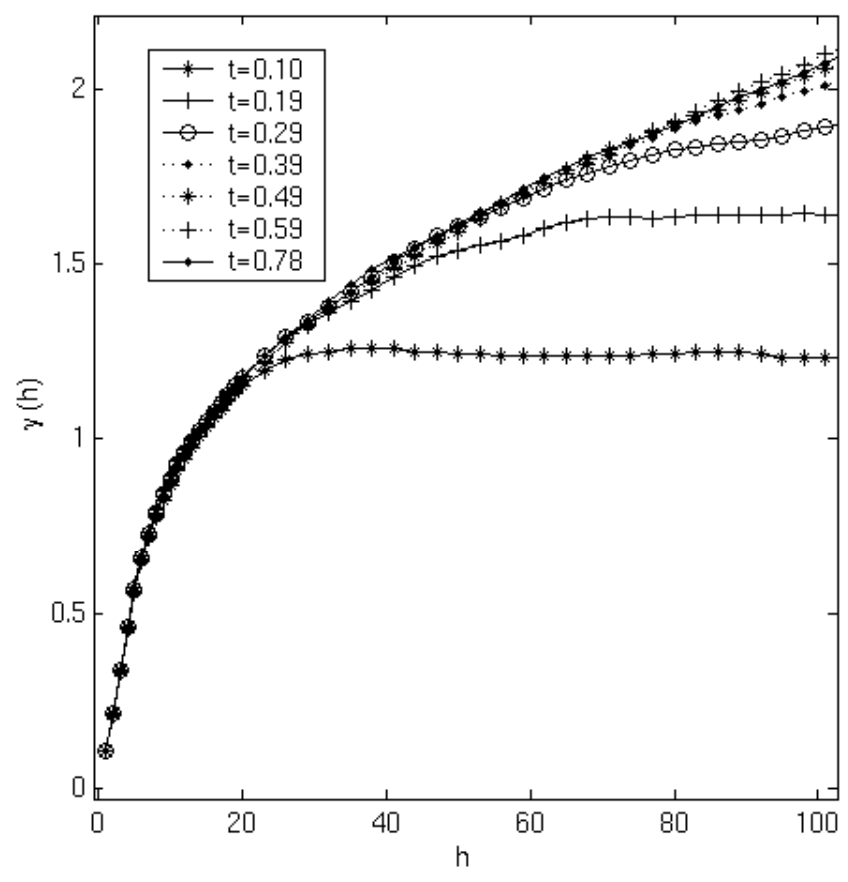

Figure 2

Evolution of the variogram of the front with adimensional time. Stable case in a isotropic medium.

long distance, due to the divergence at low-frequencies in Equation (24). The divergence occurs in 2D only: there is a complete convergence of Equation 24 at 3D. This divergence indicates that the variance characterizing the spreading of the front depends slightly on the size of the averaging volume.

As the front line is stationary, large scale saturation profiles are also stationary in the "mixing" zone. These profiles can be matched using a large-scale macrodispersion equation for the saturation, with a dispersive term acounting for the effects of the heterogeneity and a convective fractional flow term accounting for the stabilizing effect of the viscous coupling (Artus, 2003). For realistic reservoir models, statistically stationary zones in the reservoir could hence be identified and averaged with a large-scale macrodispersive term.

\section{CONCLUSION}

In order to account for the stochastic nature of current reservoir models, upscaling should be able to relate finescale random flow properties to new appropriate average properties at large scale. Sensivity tests could hence be performed directly on large-scale models, using appropriate coarse simulations grids. This approach requires the physics of two-phase flow at large scale in heterogenous media to be investigated. We have proposed a stochastic model for the dynamics of the front. This model allow the emergence of different flow regimes in the reservoir to be predicted, depending on viscosity and permeability contrasts. Using this information, an optimal coarse grid can be used for flow simulations, using large scale parameters.

\section{REFERENCES}

1 A. Ahmadi, Utilisation des propriétés équivalentes dans les modèles de réservoir : cas des écoulements diphasiques incompressibles, Thèse de Doctorat de l'Université de Bordeaux I, spécialité : Mécanique, 1992.

2 T. Arbogast and S.L. Bryant, Numerical subgrid upscaling for waterflood simulations, paper SPE 66375 Presented at the 16th SPE Symposium on Reservoir Simulation held in Houston, Texas, Feb. 11-14, 2001.

3 R. Archer, Pseudo function generation, MSc Report, Department of Petroleum Engineering of Stanford University, September 1996.

4 V. Artus, Mise à l'échelle des écoulements diphasiques dans les milieux poreux hétérogènes, Thèse de Doctorat de l'Université Paris 6 - Pierre et Marie Curie, spécialité : Géosciences et Ressources Naturelles, 2003.

$5 \mathrm{~V}$. Artus and B. Nœtinger, Macrodispersion approach for upscaling two-phase, immiscible flows in heterogeneous porous media, proc. 8th European Conference on the Mathematics of Oil Recovery, Freiberg, Germany, September 2002.

6 V. Artus, B. Notinger and L. Ricard, Dynamics of the water-oil front for two-phase, immiscible flow in heterogeneous porous media. 1-Stratified media. To appear in Transport in Porous Media, 2004.

7 K. Aziz, Reservoir simulation grids: opportunities and problems, paper SPE 25233, 12th Symposium on Reservoir Simulation, New Orleans, U.S.A., February 28-March 3, 1993.

8 J.W. Barker and S. Thibeau, A critical review of the use of pseudo-relative permeabilities for upscaling, SPE paper 35491. European 3-D Reservoir Modelling Conference, Stavanger, Norway, April 1996.

9 M. Blunt and M. Christie, How to predict viscous fingering in three component flow, Transport in porous media, 12(207-236), 1993.

10 Y.C. Chang and K.K. Mohanty, Stochastic description of multiphase flow in heterogeneous porous media, paper SPE 28443 Presented at the SPE 69th Annual Technical Conference and Exhibition, 1994.

11 K.H. Coats, R.L. Nielsen and M.H. Terhune, Simulation of three-dimensional, two-phase flow in oil and gas reservoirs, paper SPE 1961, 1967.

12 K.H. Coats, J.R. Dempsey and J.H. Henderson, The use of vertical equilibrium in two-dimensional simulation of threedimensionnal reservoir performance, SPE Journal, March 1971, pages 63-71.

13 V. Cvetkovic and G. Dagan, , Reactive transport and immiscible flow in geological media. II-Applications. Proc. Royal Society of London, 452:303-328, 1996.

14 G. Dagan, Flow and transport in porous formations. SpringlerVerlag, 1989. 
15 G. Dagan and V. Cvetkovic, Reactive transport and immiscible flow in geological media. I-General Theory. Proc. Royal Society of London, 452:285-301,1996.

16 L.P Dake, Fundamentals of reservoir engineering, Elsevier, 1978.

17 N.H. Darman, L.J. Durlofsky, K.S. Sorbie and G.E. Pickup, Upscaling immiscible gas displacements: quantitative use of fine grid flow data in grid coarsening schemes, paper SPE 59452, SPE Asia Pacific Conference on Integrated Modelling for Asset Management, Yokohama, Japan, April 2000.

18 N.H. Darman, G.E. Pickup and K.S. Sorbie, The development of an optimal grid coarsening scheme utilizing the dynamic properties of the fine-scale flow data, 8th European Conference on the Mathematics of Oil Recovery, Freiberg, Germany, 3-6 September 2002.

19 L.J. Durlofsky, Use of higher moments for the description of upscaled, process independent relative permeabilities. SPE paper 37987, SPE Reservoir Simulation Symposium, Dallas, Texas, June 1997.

20 H. Dykstra and R.L. Parsons. Secondary recovery of oil in the US, API, 1950.

21 Y.R. Efendiev and L.J. Durlofsky. Accurate subgrid models for two-phase flow in heterogeneous reservoirs. SPE paper 79680, SPE Reservoir Simulation Symposium, Houston, Texas, 3-5 February 2003.

22 S. Ekrann and J.O. Aasen, Steady-state upscaling, Transport in Porous Media, 41(245-262), December 2000

23 R.E. Fitzmorris, F.J. Kelsey and K.K. Pande, Effect of crossflow on sweep efficiency in water/oil displacement in heterogeneous reservoirs. Paper SPE 24901. $67^{\text {th }}$ Annual Technical Conference and Exhibition of the SPE, Whashington, W.D.C., 4-7 October 1992.

24 F. Furtado and F. Pereira, Scaling analysis for two-phase, immiscible flow in heterogeneous porous media. Computational and Applied Mathematics, 17(3):237-263, 1998.

25 F. Furtado and F. Pereira, Crossover from nonlinearity controlled to heterogeneity controlled mixing in two-phase porous media flows. Computational Geosciences, 7:115-135, 2003.

26 Y. Gautier, M.J. Blunt and M.A. Christie, Nested gridding and streamline-based simulation for fast reservoir performance prediction, Computational Geosciences, 3(295-320), 1999.

27 L.W. Gelhar and C.L. Axness, Three-dimensional stochastic analysis of macrodispersion in aquifers, Water Resources Research, 19-1(161-180), February 1983.

28 A. Genty, A 3D phase diagram of two-phase flow drainage regimes in porous media, Proceedings of IAMG'99, pages 699704, 1999.

29 J. Glimm, Y. Lee, Y. Ye, S. Hou and D. Sharp, Prediction of oil production with confidence intervals, Proc. SIAM Conf. on Math. and Comp. Issues in the Geosciences, 17-20 March, Austin, Texas, 2003.

30 S. Gorell and R. Bassett, Trends in reservoir simulation: Big models, scalable models? Will you please make up your mind?, paper SPE 71596, SPE Annual Technical Conference and Exhibition, New Orleans, Louisiana, 30 September-3 October 2001.
31 S.S. Guedes and D.J. Schiozer, An implicit treatment of upscaling in numerical simulation, paper SPE 51937, SPE Symposium on Reservoir Simulation, Houston 14-17 February 1999.

32 D.R. Guérillot and S. Verdière, Different pressure grids for reservoir simulation in heterogeneous reservoirs, SPE paper 29148, SPE Symposium on Reservoir Simulation, San Antonio, Texas, 12-15 February 1995.

33 R.E. Guzman, D. Giordano, F.J. Fayers, A. Godi and K. Aziz, Evaluation of dynamic pseudofunctions for reservoir simulation, SPE Journal, March 1999, pages 37-46.

34 C.L. Hearn, Simulation of stratified waterflooding by pseudorelative permeability curves. Journal of Petroleum Technology, p. 805-813, July 1971.

35 T.A. Hewett and R.A. Behrens, Scaling laws in reservoir simulation and their use in a hybrid finite difference/streamtube approach to simulating the effects of permeability heterogeneity, Reservoir Characterization, Academic Press San Diego, pages 402-441, 1989

36 H.H. Jacks, O.J.E. Smith and C.C. Mattax, The modeling of a three-dimensional reservoir with a two-dimensional reservoir simulator - The use of dynamic pseudo functions, SPE Journal, June 1973, pages 175-185.

37 K.D. Jarman, Stochastic immiscible flow with moment equations, PhD Thesis, Graduate School of the University of Colorado, 2000.

38 J.E. Killough and H.P. Foster, Reservoir simulation of the Empire Abo Field: The use of pseudos in a multilayered system, SPE Journal, October 1979, pages 279-291.

39 M.J. King and V.A. Dunayevsky, Why waterflood works: a linearized stability analysis. SPE paper 19648, 64 ${ }^{\text {th }}$ Annual Technical Conference and Exhibition held in Houston, Texas, 16-19 September 1989.

40 C.A. Kossack, J.O. Aasen and S.T. Opdal, Scaling up heterogeneities with pseudofunctions, SPE Formation Evaluation, September 1990, pages 226-389.

41 E.J. Koval, A method for predicting the performance of unstable miscible displacement in heterogeneous media, SPE Journal, June 1963, pages 145-154.

42 J.R. Kyte and D.W. Berry, New pseudo functions to control numerical dispersion. SPE 5105, pages 269-276, August 1975.

43 P. Langlo and M. Espedal, Macrodispersion for two-phase, immiscible flow in porous media. Advances in Water Resources, 17:297-316, 1994.

44 R. Lenormand, Determining flow equations from stochastic properties of a permeability field: the MHD model. SPE Journal, p. 179-190, June 1996.

45 R. Lenormand, Calculation of fractional flow for dynamic upscaling. In proceedings from the $20^{\text {th }}$ IEA Collaborative Project on enhanced Oil Recovery, 1999.

$46 \mathrm{D}$. Li and B. Beckner, Optimal uplayering for scaleup of multimillion-cell geologic models, paper SPE 62927, SPE Annual Technical Conference and Exhibition, Dallas, Texas, 1-4 October 2000.

47 J.C. Martin, Partial Integration of Equations of multiphase flow, SPE Journal, December 1968, pages 370-380. 
48 B. Notinger, Écoulements en milieux poreux hétérogènes et fracturés en régimes permanent et transitoire, Habilitation à Diriger des Recherches de l'Université Paris VI, Octobre 2000.

49 B. Noetinger, V. Artus and L. Ricard, Dynamics of the water-oil front for two-phase, immiscible flow in heterogeneous porous media. 1-Stratified media. Submitted to Transport in Porous Media, November 2002.

50 D.W. Peaceman, Calculation of transmissibilities of gridblocks defined by arbitrary corner point geometry, paper SPE 37306, 1996.

51 D.K. Ponting, Corner point geometry in reservoir simulation, Proc. 1st European Conference on the Mathematics of Oil Recovery, Cambridge, U.K., 1989.

52 M. Prevost, M.G. Edwards and M.J. Blunt, Streamline tracing on curvilinear structured and unstructured grids, SPE Journal, June 2002, pages 139-148.

53 M. Quintard and S. Whitaker, Two-phase flow in heterogeneous porous media: The method of large-scale averaging, Transport in Porous Media, 3(357-413), 1988.

54 M. Rame and J.E. Killough A New Approach to the Simulation of Flows in Highly Heterogeneous Porous Media, SPE 21247, SPE symposium on Reservoir Simulation, Anaheim 1991

55 Y. Rubin, Stochastic modelling of macrodispersion in heterogeneous porous media. Water Resources Research, 26(1):133141, January 1990.

56 P.G. Saffman and G. Taylor, The penetration of a fluid into a porous medium or Hele-Shaw cell containing a more viscous fluid. Proc. Royal Society of London, A245:312-329, 1958.

57 A.D. Simon and L.F. Koederitz, An improved method for the determination of pseudo-relative permeability data for stratified systems. SPE paper 10975, Annual Fall Technical Conference and Exhibition, New-Orlean, L.A., September 1982.

58 D. Stern and A.G. Dawson, A technique for generating reservoir simulation grids to preserve geologic heterogeneity, paper SPE 51942, SPE Reservoir Simulation Symposium, Houston, Texas, 14-17 February 1999.
59 W.M. Stiles, Use of permeability distribution in waterflood calculations. Trans. A.I.M.E. p. 9-13, January 1949.

60 H.L. Stone, Rigorous black oil pseudo functions, paper SPE 21207, 11th SPE Symposium on Reservoir Simulation, Anaheim, California, 1991.

61 M.R. Todd and W.J. Longstaff, The development, testing and application of a numerical simulator for predicting miscible flood performance, T. AIME, 253(874-882), 1972.

62 S. Verdière and D.R. Guérillot, Dual mesh method for multiphase flows in heterogeneous media, 5th European Conference on the Mathematics of Oil Recovery, Leoben, Austria, 3-6 September 1996.

63 S. Verdière and M.H Vignal, Numerical and theoretical study of a dual mesh method using finite volume schemes for twophase flow problems in porous media, Numerical Mathematics, 80(601-639), 1998.

64 Y. Yokoyama and L.W. Lake, The effects of capillary pressure on immiscible displacements in stratified porous media, paper SPE 10109, 56th Annual Fall Meeting of the SPE, San Antonio, October 1981.

65 Y.C. Yortsos, Analytical studies for processes at vertical equilibrium. SPE paper 26022, 1992.

66 V.J. Zapata and L.W. Lake, A theoretical analysis of viscous crossflow. Paper SPE 10111. 56 ${ }^{\text {th }}$ Annual Fall Technical Conference and Exhibition of the SPE, San Antonio, Texas, 3-7 October 1981.

67 D. Zhang, Stochastic methods for flow in porous media, Academic Press, 2001.

68 D. Zhang, L. Li and H.A. Tchelepi, Stochastic formulation for uncertainty assessment of two-phase flow in heterogeneous reservoirs. SPE paper 51930, SPE Reservoir Simulation and Symposium held in Houston, Texas, February 1999.

Manuscript received in January 2004 